13

\title{
Разрушение пептидов и нуклеозидов в реакциях с электронами низких энергий
}

\author{
(C) М.В. Мусртахов, П.В. Щукин
}

Институт фризики молекул и кристаллов Уфимского научного центра $\mathrm{AH}$, 450075 Уфра, Россия

e-mail: LMSNI@anrb.ru

(Поступило в Редакцию 11 июля 2017 г.)

Методом масс-спектрометрии отрицательных ионов исследованы процессы диссоциативного захвата электронов молекулами некоторых нуклеозидов, простейших ди- и трипептидов, модифицированных дипептидов. Для исследованных объектов установлены энергетические области и эффективность диссоциативного захвата электронов, оценены пороговые энергии некоторых фрагментационных процессов. Обнаружено, что цитидин и пептиды устойчивы к фрагментации простым разрывом связей в диапазоне энергии электронов $\sim 0-1 \mathrm{eV}$.

DOI: 10.21883/JTF.2018.05.45907.2425

\section{Введение}

На сегодняшний день биомолекулярная электроника является бурно развивающимся перспективным направлением наноэлектроники, где в качестве компонентов устройств применяются отдельные биомолекулы и их ансамбли [1]. Среди наиболее перспективных органических материалов здесь можно выделить молекулы ДНК и пептидов, электрическая проводимость которых может изменяться в широких пределах [2,3]. В отличие от обычных органических молекул биомолекулы обладают важными для создания электронных устройств свойствами: самовоспроизводимость, возможность копирования и самосборки. Так, в настоящее время можно легко синтезировать молекулу ДНК с нужной последовательностью нуклеотидов длиной в несколько тысяч звеньев. Также из молекул ДНК возможно построение не только линейной цепочки, но и дву- и трехмерной сетки или еще более сложных пространственных объектов [4]. Среди преимуществ использования органических биоматериалов в сравнении с традиционными полупроводниками легкость, механическая гибкость, энергоэффективность, низкая стоимость и простота производства. В отличие от обычных кристаллических полупроводников полупроводники из ДНК не токсичны и хорошо разлагаются под действием микроорганизмов, не нанося вред окружающей среде [5]. Многочисленные исследования показывают, что на основе молекул ДНК и пептидов может быть создан широкий круг электронных компонентов: нанотранзисторы, нанодиоды, логические элементы, элементы памяти, солнечные батареи и другие приборы нанометрового масштаба [6]. Таким образом, эти соединения обладают большим потенциалом для использования в современной электронике.

Среди основных требований, предъявляемых к таким материалам, являются их устойчивость по отношению к внешним воздействиям - агрессивной среде (вода, кислород, кислоты и пр.), электромагнитному облучению, перепадам температуры, механическим нагрузкам, давлению и пр. Одним из малоизученных, но важных аспектов стабильности материалов является их устойчивость к воздействию низкоэнергетических электронов, возникающих при внутреннем фотоэффекте или протекании через них электрического тока и приводящих к диссоциации межатомных связей часто даже без значительных энергетических затрат. В этом случае распад молекулярных систем обусловлен реакциями резонансного присоединения электронов к молекулам. В результате этих реакций возникают метастабильные образования - временноживущие отрицательные ионы (ОИ), дальнейшая эволюция которых приводит к распаду по доступным каналам фрагментации или спонтанному выбросу добавочного электрона. Основным методом исследований в этом направлении является эксперимент, для проведения которого хорошо подходит масс-спектрометрия отрицательных ионов, образующихся вследствие резонансного захвата электронов. Этим методом в работе были исследованы некоторые нуклеозиды и олигопепептиды.

\section{1. Экспериментальная часть}

Эксперимент выполнен на магнитном массспектрометре МИ-1201В (Сумы, Украина), модифицированном для работы с ОИ [7]. Вкратце, электроны, эмитируемые катодом, проходят через камеру ионизации, где взаимодействуют с парами образцов, что приводит к образованию ОИ. Последние извлекаются из камеры, формируются в пучок, ускоряются, массанализируются, регистрируются вторично-электронным умножителем. Управление энергией электронного пучка осуществляется компьютером, в который синхронно заносится регистрируемый ионный сигнал. Для калибровки шкалы электронной энергии мы использовали максимумы кривых эффективного выхода ионов $\mathrm{SF}_{6}^{-} / \mathrm{SF}_{6}$ $(\sim 0 \mathrm{eV})$ и $[\mathrm{M}-\mathrm{H}]^{-} / \mathrm{CH}_{3} \mathrm{COOH}(\sim 1.55 \mathrm{eV}[8])$. Энергии появления ионов определялись относительно энергии 
<smiles>O=c1cc[nH]c(=O)[nH]1</smiles>

Uracil (I)<smiles>O=c1ccn(C2OC(CO)C(O)C2O)c(=O)[nH]1</smiles>

Uridine (VII)<smiles>Nc1ncnc2[nH]cnc12</smiles>

Adenine (II)<smiles></smiles>

Adenosine (VIII)<smiles>Nc1cc[nH]c(=O)n1</smiles>

Cytosine (III)<smiles>Nc1ccn(C2OC(CO)C(O)C2O)c(=O)n1</smiles>

Cytidine (IX)<smiles>CC1CNC(=O)NC1=O</smiles>

Thymine (IV)<smiles>Cc1cn(C2CC(O)C(CO)O2)c(=O)[nH]c1=O</smiles>

Thymidine (X)<smiles>OCC1OC(O)C(O)C1O</smiles>

Ribose (V)<smiles>OCC1COC(O)C1</smiles>

Deoxyribose (VI)<smiles>O=C1CN(C2CC(O)CO2)C(=O)N1</smiles><smiles></smiles><smiles></smiles>

Deoxyuridine (XI) Deoxyadenosine (XII) Deoxycitidine (XIII)<smiles>NCC(=O)NCC(=O)O</smiles><smiles>CC(N)C(=O)NC(C)C(=O)O</smiles><smiles>CC(CC(=O)NCC(=O)NCC(=O)O)NCCN</smiles><smiles>CSCCC(N)C(=O)O</smiles><smiles>NC(CS)C(=O)O</smiles>

Diglycine (XVII)

Dialanine (XIX)

Diglycylalanine (XX)<smiles>CC(NC(=O)CN)C(=O)O</smiles><smiles>CC(CS)C(=O)N1CCCC1C(=O)O</smiles>

Glycylalanine (XVIII) Captopril (XXIII)<smiles>CC(NC(=O)C(C)NC(C)C(=O)O)C(=O)O</smiles>

Trialanine (XXI)<smiles>COC(=O)C(N)CS</smiles>

Cysteine methyl ester (XVI)

Aspartame (XXII) появления $\mathrm{SF}_{6}^{-} / \mathrm{SF}_{6}(\sim 0 \mathrm{eV})$. Разрешение по энергии электронов было $\Delta E_{1 / 2}=0.5-0.6 \mathrm{eV}$.

Образцы соединений приобретены в Sigma/Aldrich Chemical Co. и исследовались без дополнительной очистки. Для напуска паров серосодержащих соединений (цистеин, метионин, метиловый эфир цистеина, каптоприл) в камеру ионизации применялась трубка прямого ввода, подогреваемая до температуры оптимальной сублимации вещества. При этом температура камеры поддерживалась на $10-30^{\circ}$ выше. Для остальных соединений использовалась другая методика, при которой образец заранее помещался на дно камеры ионизации, откуда происходило его испарение в результате нагрева. Эта методика в отличие от использования трубки прямого ввода твердых образцов позволяет получать необходи- мое давление паров в камере при сравнительно низких температурах нагрева, что очень важно при работе с пептидами и нуклеозидами. На схеме представлены структурные формулы исследованных объектов.

\section{2. Расчеты}

Энергетический порог диссоциативных реакций вычислялся на основе энтальпий образования нейтральных частиц и ионов, полученных путем корректировки нейронным методом X1 [9] полных энергий частиц, рассчитанных методом функционала плотности B3LYP в базисе $6-311+\mathrm{G}(\mathrm{d}, \mathrm{p})$. 

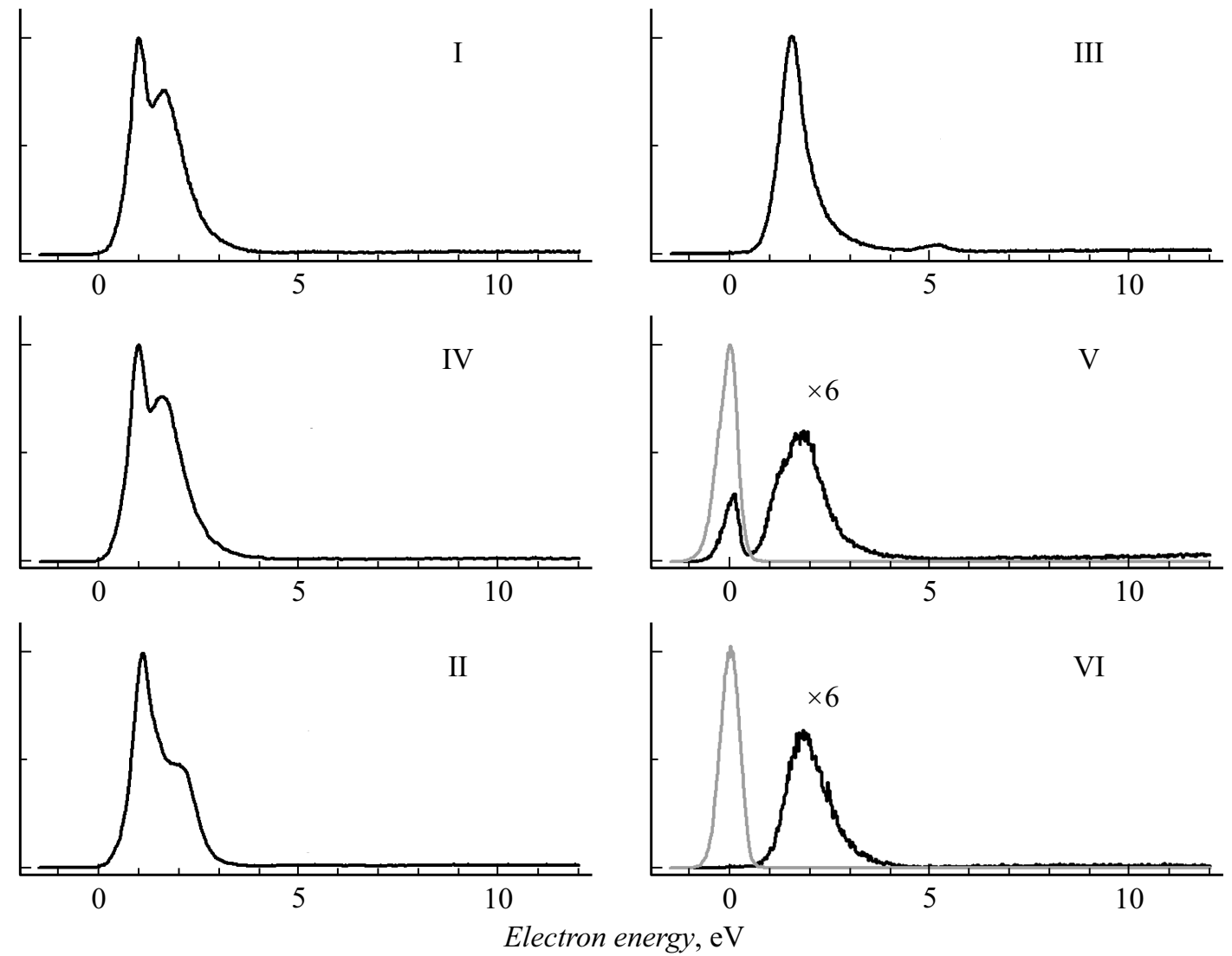

Рис. 1. Кривые эффективного выхода ионов $[\mathrm{M}-\mathrm{H}]^{-}$из азотистых оснований и сахаров (черная линия) в зависимости от электронной энергии при температурах: $119^{\circ} \mathrm{C}$ (тимин), $121^{\circ} \mathrm{C}$ (урацил), $103^{\circ} \mathrm{C}$ (аденин), $140^{\circ} \mathrm{C}$ (цитозин), $82^{\circ} \mathrm{C}$ (рибоза), $60^{\circ} \mathrm{C}$ (дезоксирибоза). Для сахаров показаны кривые эффективного выхода основных ионов с $m / z 132$ в масс-спектре рибозы и $m / z, 98$ в масс-спектре дезоксирибозы (серая линия).

\section{3. Результаты и обсуждение}

\section{1. Нуклеозиды}

Нуклеозиды состоят из фрагментов азотистого основания и сахара (рибозы или дезоксирибозы) и в некоторой степени должны обладать их свойствами. Пик ионов $[\mathrm{M}-\mathrm{H}]^{-}$в диапазоне энергии $1-4 \mathrm{eV}$ является основным в масс-спектрах азотистых оснований [10-17], и кривые эффективного выхода для четырех из них представлены на рис. 1. Доминирующим по интенсивности в масс-спектре рибозы является пик в области тепловых энергий $(\sim 0 \mathrm{eV}) \mathrm{c} m / z 132$, отвечающий ионам $\left[\mathrm{M}-\mathrm{H}_{2} \mathrm{O}\right]^{-\bullet}$, а в масс-спектре дезоксирибозы пик в области тепловых энергий с $m / z 98$, отвечающий ионам $\left[\mathrm{M}-2 \mathrm{H}_{2} \mathrm{O}\right]^{-\bullet}[18]$. Кривые эффективного выхода для них и для ионов $[\mathrm{M}-\mathrm{H}]^{-}$также представлены на рис. 1. Ранее ОИ этих сахаров были исследованы в работах [19-21].

Резонансный захват электронов молекулами тимидина исследовался ранее, и в масс-спектре были зарегистрированы три пика ионов, соответствующие процессам выброса атома водорода, фрагмента сахара и молекулы основания $[22,23]$. Позже нами был получен масс-спектр
ОИ уридина, включающий 16 пиков ионов [24]. Дополнительно к уридину мы исследовали процессы образования ОИ еще из нескольких соединений. Формально синтез нуклеозидов из молекулы азотистого основания и молекулы сахара сопровождается выделением молекулы воды. Согласно результатам [23], возможен и обратный процесс - терморазложение нуклеозидов на сахар и основание при взаимодействии с водой, присутствующей в образцах. Для контроля этого процесса мы регистрировали характеристичные для масс-спектров рибозы или дезоксирибозы пики ионов.

На рис. 2 для семи объектов представлен полный ионный ток в функции от электронной энергии, полученный суммированием кривых эффективного выхода всех осколочных ОИ. Распад молекулярных ионов тимидина, уридина, дезоксиуридина и аденозина в области энергии ниже $5 \mathrm{eV}$ осуществляется по меньшему числу каналов, чем в области энергии свыше $5 \mathrm{eV}$, и с меньшей эффективностью. Основной вклад в полный ионный ток здесь вносят процессы выброса фрагмента сахара и атома водорода. Основной пик в масс-спектрах нуклеозидов соответствует ионам $[\mathrm{M}-\mathrm{Caxap}]^{-}$в высокоэнергетической области. Относительная интенсивность пиков ионов с $m / z, 132$ или с $m / z, 98$ в масс-спектрах не пре- 

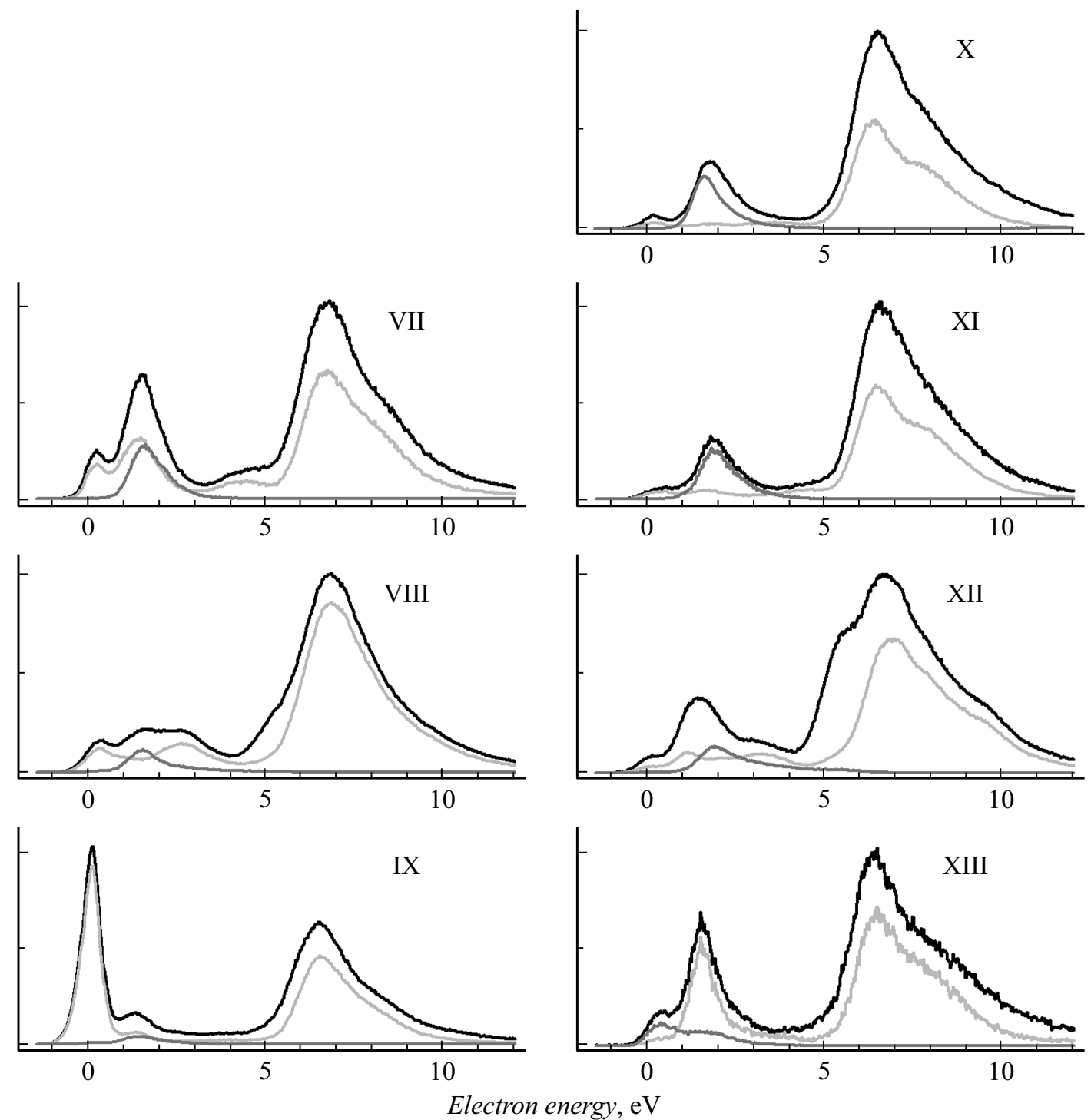

Рис. 2. Суммарные кривые эффективного выхода ОИ (черная линия), ионов $[\mathrm{M}-\mathrm{H}]^{-}$(темно-серая линия) и ионов $[\mathrm{M}-\mathrm{Caxap}]^{-}$ (светло-серая линия) из нуклеозидов в зависимости от электронной энергии при температурах: $130^{\circ} \mathrm{C}$ (уридин), $220^{\circ} \mathrm{C}($ аденозин), $160^{\circ} \mathrm{C}$ (цитидин), $138^{\circ} \mathrm{C}$ (тимидин), $125^{\circ} \mathrm{C}$ (дезоксиуридин), $168^{\circ} \mathrm{C}$ (дезоксиаденозин), $154^{\circ} \mathrm{C}$ (дезоксицитидин).

вышала $1 \%$, что свидетельствовало о незначительности термодеструкции веществ из-за взаимодействия с водой или же ее отсутствии, так как рибоза или дезоксирибоза могли присутствовать в образцах в виде примесей.

В масс-спектре дезоксиаденозина мы регистрировали пик ионов с $m / z 98$ с относительной интенсивностью $\sim 12 \%$, а на кривой эффективного выхода ионов $[\mathrm{M}-\mathrm{Caxap}]^{-}$был зарегистрирован острый пик с максимумом в районе энергии $1.1 \mathrm{eV}$ с относительной интенсивностью $\sim 15 \%$, что соответствует ионам $[\mathrm{M}-\mathrm{H}]^{-}$из аденина (рис. 1). Эти факты свидетельствуют о частичном терморазложении нуклеозида. (Заметим, что коммерческий образец изначально содержал воду, поскольку вещество представляет собой моногидрат дезоксиаденозина. Для удаления воды его пришлось длительно прогревать в вакууме с постепенным повышением тем- пературы.) Более драматичная картина наблюдалась при исследовании дезоксицитидина. На кривой эффективного выхода ионов $[\mathrm{M}-\mathrm{Caxap}]^{-}$зарегистрирован острый пик с максимумом в районе энергии $1.5 \mathrm{eV}$ с относительной интенсивностью 74\%, что соответствует ионам $[\mathrm{M}-\mathrm{H}]^{-}$ из цитозина (рис. 1), а относительная интенсивность пика ионов с $m / z 98$ составляла 51\%. Этот результат очень напоминает полученный для тимидина в работе [23], и мы также провели эксперимент при разных температурах с регистрацией характеристичных ионов.

Повышение температуры вызвало рост давления паров образца дезоксицитидина, и, как следствие, увеличилась абсолютная интенсивность ионов (рис. 3). Относительная интенсивность пика при $1.5 \mathrm{eV}$ на кривой выхода ионов $[\mathrm{M}-\mathrm{Caxap}]^{-}$увеличилась в $\sim 8$ раз, что коррелирует с изменением относительной интенсивно- 


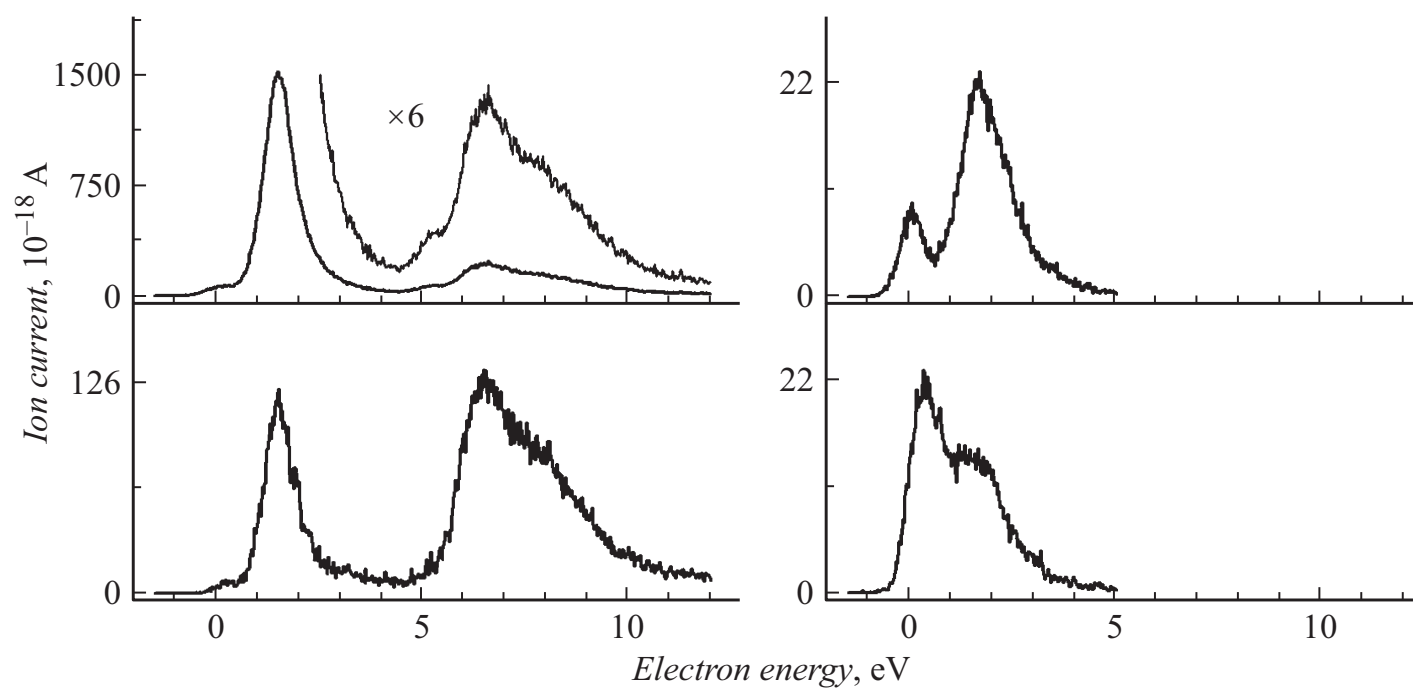

Рис. 3. Кривые эффективного выхода ионов $[\mathrm{M}-\mathrm{Caxap}]^{-}$(слева) и ионов $[\mathrm{M}-\mathrm{H}]^{-}$(справа) из дезоксицитидина в зависимости от электронной энергии, полученные при температурах $169^{\circ} \mathrm{C}$ (верхние панели) и $156^{\circ} \mathrm{C}$ (нижние панели); относительная интенсивность пика ионов с $m / z 98$ в масс-спектре составляет $1100 \%$ для высокой температуры и $61 \%$ для низкой температуры.

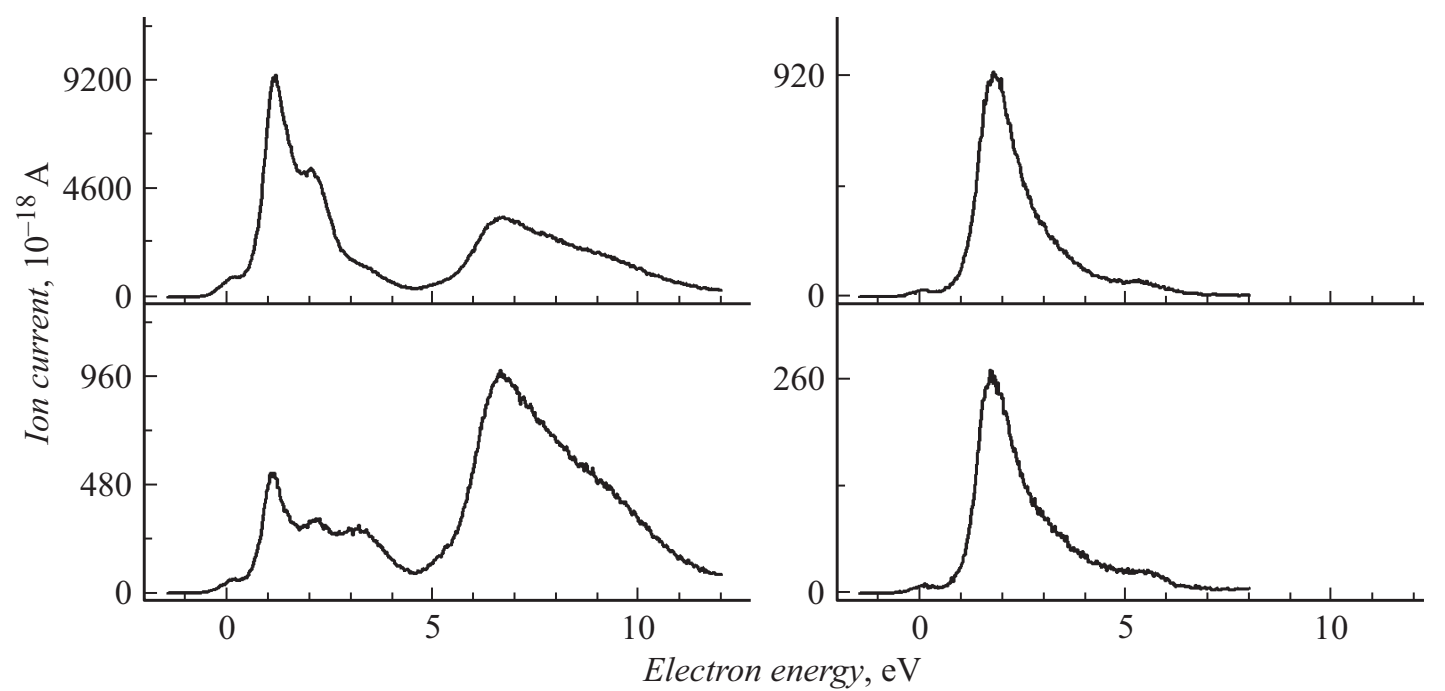

Рис. 4. Кривые эффективного выхода ионов $[\mathrm{M}-\mathrm{Caxap}]^{\circ}$ (слева) и ионов $[\mathrm{M}-\mathrm{H}]^{-}$(справа) из дезоксиаденозина в зависимости от электронной энергии, полученные при температурах $162^{\circ} \mathrm{C}$ (верхние панели) и $141^{\circ} \mathrm{C}$ (нижние панели); относительная интенсивность пика ионов с $\mathrm{m} / \mathrm{z} 98$ в масс-спектре составляет 76\% для высокой температуры и $17 \%$ для низкой температуры.

сти пика ионов с $m / z$ 98. Этот эксперимент (как и данные работы [24]) показывает, что нагрев нуклеозида в присутствии воды приводит к его разложению на сахар и основание. Отметим, что относительная интенсивность пика при тепловых энергиях также возросла, но его источником не мог быть образующийся цитозин (рис. 1). Наблюдалось изменение формы кривой эффективного выхода ионов $[\mathrm{M}-\mathrm{H}]^{-}$, проявившееся в уменьшении абсолютной интенсивности пика при тепловых энергиях. Мы заключили, что источником этого пика является легко летучая примесь в образце. Эксперимент с вариацией температуры мы провели также с дезоксиаденозином без предварительной просушки образца, и его результаты приведены на рис. 4. Подобно результатам для дезоксицитидина, при повышении температуры мы наблюдали изменение формы кривой эффективного выхода ионов $[\mathrm{M}-\mathrm{Caxap}]^{-}$, но форма кривой эффективного выхода ионов $[\mathrm{M}-\mathrm{H}]^{-}$и их относительная интенсивность в диапазоне энергии 0-7 eV не изменялась.

Цитидин немного выбивается из общей картины ионообразования в нуклеозидах благодаря интенсивному пику ионов $[\mathrm{M}-\mathrm{Caxap}]^{-}$в области тепловых энергий (рис. 2). Вероятно, этот пик не является результатом разложения нуклеозида на сахар и основание, поскольку в масс-спектре относительная интенсивность пика ионов с $m / z 132$ составляла 1.7\%. Чтобы выяснить его 


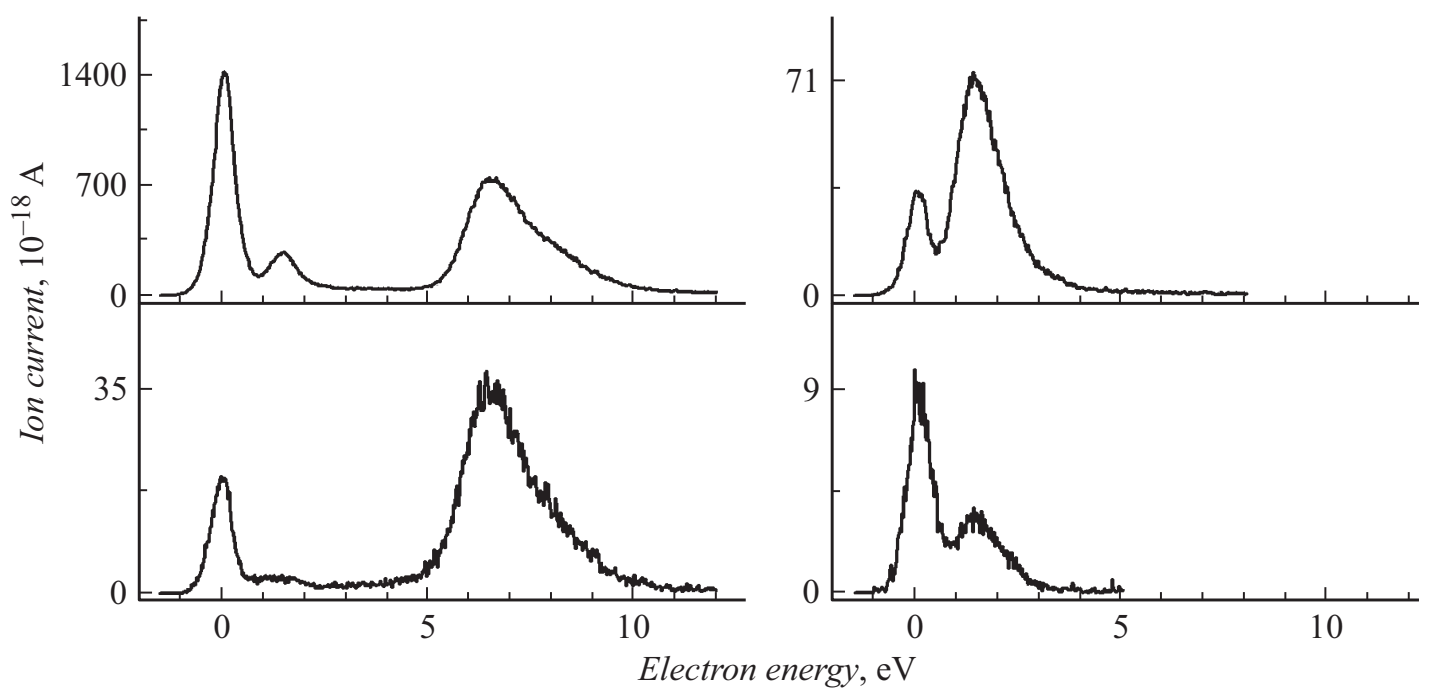

Рис. 5. Кривые эффективного выхода ионов $[\mathrm{M}-\mathrm{Caxap}]^{-}$(слева) и ионов $[\mathrm{M}-\mathrm{H}]^{-}$(справа) из цитидина в зависимости от электронной энергии, полученные при температурах $162^{\circ} \mathrm{C}$ (верхние панели) и $141^{\circ} \mathrm{C}$ (нижние панели); относительная интенсивность пика ионов с $\mathrm{m} / z 132$ в масс-спектре составляет 51\% для высокой температуры и $14 \%$ для низкой температуры.

происхождение, мы провели эксперимент с вариацией температуры, причем цитидин исследовался вместе с дезоксиаденозином. Последний был необходим для достижения и контроля стабильности условий эксперимента, поскольку низкий выход ионов из цитидина при $T=141^{\circ} \mathrm{C}$ не позволял выполнить эту процедуру корректно. Отметим, что характеристичные ионы $[\mathrm{M}-\mathrm{H}]^{-}$ и $[\mathrm{M}-\mathrm{Caxap}]^{-}$из цитидина не имеют ,двойников“" $\mathrm{c}$ $\mathrm{m} / z 242$ и $\mathrm{m} / \mathrm{z} 110$ в масс-спектре дезоксиаденозина; то же самое справедливо и для характеристичных ионов с $m / z 250$ и $m / z 134$ из дезоксиаденозина.

При повышении температуры существенно увеличилась абсолютная интенсивность ионов в масс-спектре цитидина (рис. 5). Как и в эксперименте с дезоксицитидином, форма кривой эффективного выхода ионов $[\mathrm{M}-\mathrm{H}]^{-}$изменилась, но уменьшилась лишь относительная интенсивность пика при тепловых энергиях. Это могло быть связано с примесями в образце или же другими причинами. Обнаружилось также изменение формы кривой выхода ионов $[\mathrm{M}-\mathrm{Caxap}]^{-}$в диапазоне энергии 0-2.5 eV. Рост относительной интенсивности пика при $1.5 \mathrm{eV}$ мы связываем с разложением нуклеозида на основание и сахар при взаимодействии с водой, как это было в случае дезоксицитидина.

Для обнаруженной зависимости от температуры пика при тепловых энергиях может быть несколько альтернативных причин, одна из которых предполагает терморазложение без участия воды. Другой причиной может быть эффект „горячей полосы“, который отражает больцмановское распределение населенности возбужденных колебательных уровней молекул. Согласно квантовохимическим расчетам, пороговая энергия процесса выброса фрагмента рибозы из молекулярного иона составляет $0.42 \mathrm{eV}$, т. е. для электронов с энергией $\sim 0 \mathrm{eV}$ недостаток энергии $(\Delta E)$ для протекания реакции составляет
$0.42 \mathrm{eV}$. Но расчет по формуле Больцмана [25] для изменения относительной интенсивности пика ионов в 3.5 раза при изменении температуры со $141^{\circ} \mathrm{C}$ до $162^{\circ} \mathrm{C}$ дает значение $\Delta E=1.08 \mathrm{eV}$. Возможно, что завышение $\Delta E$ на $0.66 \mathrm{eV}$ обусловлено возникновением энергетического барьера в результате „пересечения“ потенциальных поверхностей начального и конечного состояний молекулярных ионов в процессе предиссоциации [10]. Рис. 6 отчасти поддерживает эту гипотезу. Здесь показано схематическое разделение двух пиков на кривой эффективного выхода ионов $[\mathrm{M}-\mathrm{Caxap}]^{-}$посредством вычитания из нее нормированной сглаженной кривой выхода постороннего иона с $m / z 137$ (интенсивность последнего низкая, но форма кривой почти идеально совпадает с таковой для иона $[\mathrm{M}-\mathrm{Caxap}]^{-}$в области энергии $<1 \mathrm{eV})$. Результатом этой процедуры является резонансный пик с максимумом в районе $1.4 \mathrm{eV}$ и энергией появления $\sim 1 \mathrm{eV}$. На этом же рисунке приведена кривая эффективного выхода ионов $[\mathrm{M}-\mathrm{H}]^{-}$, на которой второй пик имеет схожие параметры - максимум наблюдается при $1.4 \mathrm{eV}$, а измеренная энергия появления составляет $\sim 1 \mathrm{eV}$. Третьей причиной зависимости от температуры пика ионов $[\mathrm{M}-\mathrm{Caxap}]^{-}$при тепловых энергиях может быть существование изомерной формы цитидина, в которой фрагмент основания представляет иминную структуру. Для этой формы пороговая энергия обсуждаемого процесса составляет $0.05 \mathrm{eV}$. Но расчеты по формуле Больцмана для концентрации этой формы в смеси изомеров при низкой и высокой температурах эксперимента дают значения 4.8 и 5.6\% соответственно, и изменение концентрации не согласуется с изменением относительной интенсивности пика ионов. В связи с существованием изомерных форм цитидина возможен вариант прегруппировочной реакции с выделением обсуждаемых ионов при тепловых энергиях. То есть элек- 


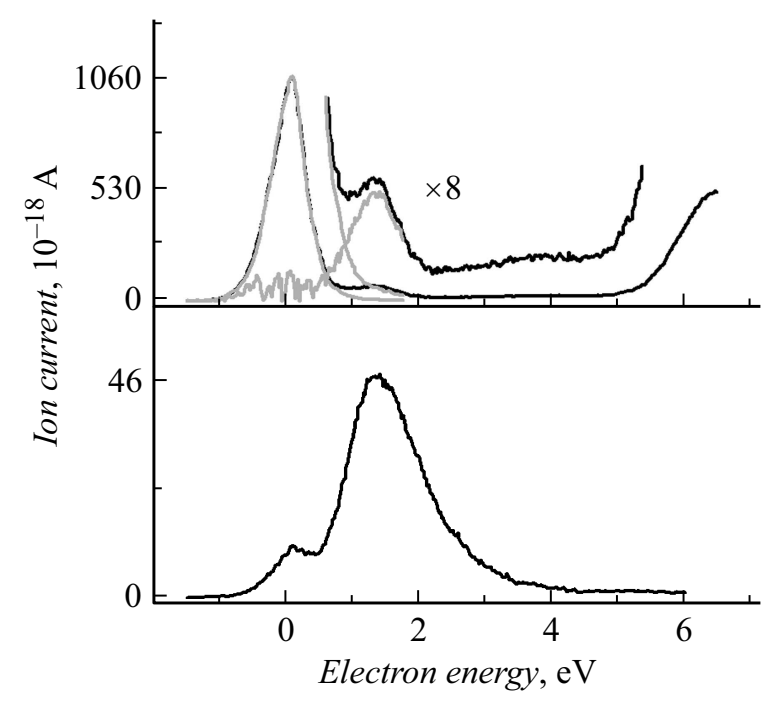

Рис. 6. Фрагменты кривых эффективного выхода ионов $[\mathrm{M}-\mathrm{Caxap}]^{-}$(верхняя панель) и ионов $[\mathrm{M}-\mathrm{H}]^{-}$(нижняя панель) из цитидина в зависимости от электронной энергии, взятые из рис. 2; относительная интенсивность пика ионов с $m / z 132$ в масс-спектре составляет 1.7\%. На верхней панели серыми линиями показаны: сглаженная нормированная кривая эффективного выхода ионов с $m / z 137$ из примеси с максимумом в районе $\sim 0 \mathrm{eV}$ и как результат вычитания последней из экспериментальной кривой для ионов $[\mathrm{M}-\mathrm{Caxap}]^{-}-$ выделенный резонансный пик с максимумом в районе $\sim 1.4 \mathrm{eV}$.

троны захватываются молекулами с аминной структурой фрагмента основания, а ионы $[\mathrm{M}-\mathrm{Caxap}]^{-}$выделяются с иминной структурой. Пороговая энергия для этого процесса составляет $\sim 0.16 \mathrm{eV}$, что не позволяет ему протекать нормальным образом при тепловых энергиях электронов, как и в выше обсужденных случаях.

Анализ полученных данных свидетельствует о том, что нуклеозиды легко разлагаются на азотистое основание и сахар при нагреве в присутствии воды. Как показали эксперименты, этот процесс зависит от содержания воды в образце, и при комнатной или чуть большей температуре он должен происходить намного медленнее, чем в условиях проведенных экспериментов. Вода основа органической жизни и в то же время она является ее разрушителем. В ходе эволюции живые организмы выработали некий защитный механизм против спонтанного распада нуклеиновых кислот, но процесс разрушения ДНК- и РНК-элементов в микроэлектронных устройствах от действия влаги, по-видимому, является лишь вопросом времени. Фрагментация ионизованных форм большинства нуклеозидов происходит во всем диапазоне энергии, начиная с $\sim 0 \mathrm{eV}$. Исключением является цитидин, который фрагментирует, начиная с $\sim 1 \mathrm{eV}$. Возможно, что к такому же исключению относится и дезоксицитидин, но подтвердить экспериментально это предположение не представлялось возможным из-за частичного разложения объекта. С целью выяснения стабильности к фрагментации нуклеиновых кислот при протекании по
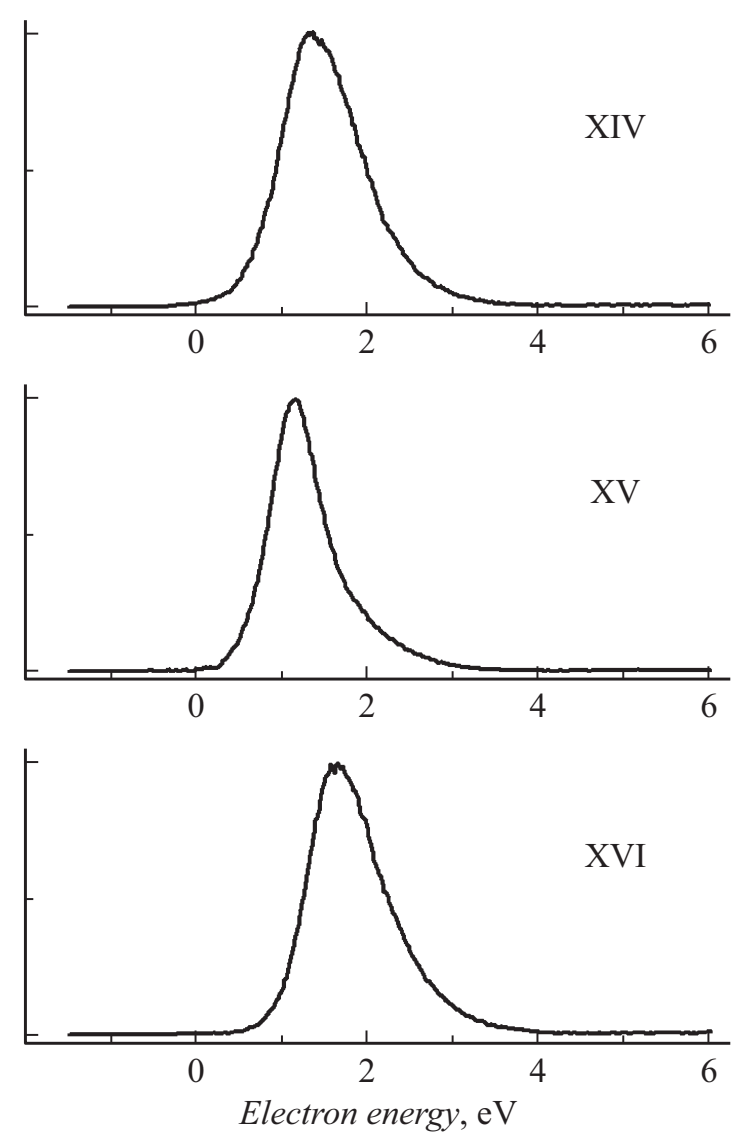

Рис. 7. Кривые эффективного выхода ионов $[\mathrm{M}-\mathrm{H}]^{-}$в зависимости от электронной энергии из цистеина $\left(T=92^{\circ} \mathrm{C}\right)$, метионина $\left(T=135^{\circ} \mathrm{C}\right)$ и метилового эфира цистеина $\left(T=31^{\circ} \mathrm{C}\right)$.

ним электрического тока более информативным было бы исследование образования ОИ из нуклеотидов и фосфатов сахаров. Мы провели эксперименты с $5^{\prime}$ монофосфатом цитидина, 5'-монофосфатом уридина и $6^{\prime}$ фосфатом глюкозамина, но неудачно. Вещества не удалось испарить даже при сильном нагреве, и мы регистрировали пики характеристичных ионов для нуклеозидов, азотистых оснований, сахаров и фосфорной кислоты, которые свидетельствовали о терморазложении. Такие труднолетучие и легко разлагающиеся объекты исследуют с помощью методов электроспрей и MALDI. Например, последним в работе [26] проводилось комплексное исследование ОИ из тимидинсодержащего нуклеотида и его компонентов. Но, к сожалению, эти методы не дают информации об энергетике распадных процессов.

\section{2. Пептиды}

Поликонденсация аминокислот в пептиды сопровождается выделением молекулы воды, состоящей из ОНгруппы карбоксильного фрагмента одной аминокислоты и Н-атома аминогруппы другой аминокислоты с образованием пептидной группы [27]. Последняя обусловливает новые специфические свойства молекул пептидов, но 
при этом сохраняются некоторые свойства аминокислот, благодаря наличию концевых $\mathrm{COOH}-$ и $\mathrm{NH}_{2}$-групп, а также боковых заместителей фрагментов аминокислот в пептиде. Одно из таких свойств проявляется в интенсивной фрагментации короткоживущих молекулярных ОИ аминокислот с выбросом атома водорода СООН-группы в низкоэнергетической области. Пик ионов $[\mathrm{M}-\mathrm{H}]^{-}$в диапазоне энергии $1-3 \mathrm{eV}$ является основным в массспектрах аминокислот [28-35], а относительная интенсивность пиков других ионов не превышает 10\%. Другое свойство аминокислот проявляется в перегруппировочных реакциях с участием Н-атома СООН-группы с выбросом частиц $\mathrm{NH}_{2}^{\bullet}$ и $\mathrm{NH}_{3}$ (и, возможно, с выбросом бокового заместителя в фенилаланине, тирозине, триптофане - наши неопубликованные данные) в указанной области энергии. Для ряда аминокислот, по-видимому, характерна фрагментация молекулярных ионов в результате потери атома водорода из заместителя, например, для аспарагиновой и глутаминовой кислот, в боковом заместителе которых есть карбоксильная группа. В литературе нет прямых доказательств этой гипотезы, но эксперименты с метионином и метиловым эфиром цистеина свидетельствуют о том, что кривая эффективного выхода ионов $[\mathrm{M}-\mathrm{H}]^{-}$из цистеина обусловлена процессами выброса Н-атомов из карбоксильной и тиоловой групп (рис. 7) [36]. Вероятно, такое же происхождение имеют ионы $[\mathrm{M}-\mathrm{H}]^{-}$из тирозина и триптофана, поскольку в метиловых эфирах этих объектов регистрировали ионы $[\mathrm{M}-\mathrm{H}]^{-}$в районе энергии $1.3-1.6 \mathrm{eV}[33]$.

ОИ из ди- и трипептидов, состоящих из фрагментов молекул глицина и аланина, были исследованы нами ранее [37-40]. На рис. 8 представлен полный ионный ток в функции от электронной энергии для каждого объекта, полученный суммированием кривых эффективного выхода всех ОИ. Распад молекулярных ионов в области энергии ниже $4 \mathrm{eV}$ осуществляется по меньшему числу каналов, чем в области энергии свыше $4 \mathrm{eV}$, но с большей эффективностью. Основной вклад в полный ионный ток здесь вносят процессы выброса карбоксильного атома водорода и перегруппировочные реакции, обусловленные миграцией этого Н-атома. На этом же рисунке представлены кривые эффективного выхода ионов, образовавшихся простым разрывом $\mathrm{C}_{\alpha}-\mathrm{N}$ связи: для дипептидов - ионы $\mathrm{NH}_{2} \mathrm{CH}(\mathrm{R}) \mathrm{CONH}^{-}$, для трипептидов - суммарная кривая выхода ионов $\mathrm{NH}_{2} \mathrm{CH}(\mathrm{R}) \mathrm{CONH}^{-}$и $\mathrm{NH}_{2} \mathrm{CH}(\mathrm{R}) \mathrm{CONHCH}(\mathrm{R}) \mathrm{CONH}^{-}$ $\left(\mathrm{R}=\mathrm{H}\right.$ или $\left.\mathrm{CH}_{3}\right)$. Измеренные энергии появления перечисленных ионов составляют $1.0-1.4 \mathrm{eV}$, что согласуется с расчетами пороговых энергий этих реакций, например, для диглицина расчет дает значение $1.04 \mathrm{eV}$.

При исследовании модифицированного дипептида аспартама было установлено, что большинство ионов в области низких энергий образуются выбросом атома водорода из карбоксильной группы бокового заместителя фрагмента молекулы аспарагиновой кислоты и в результате перегруппировочных реакций с участием этого Н-атома [41]. Для этого объекта на рис. 8 представлен полный ионный ток в функции от электронной энергии и суммарная кривая эффективного выхода ионов с $m / z 131$ и $m / z 59$. В кривую выхода ионов с $m / z 131$ могут вносить вклад два процесса - перегруппировочная реакция (1) с выделением иона карбоксильной структуры при меньшей энергии и реакция (2) простого разрыва связи $\mathrm{N}-\mathrm{C}_{\alpha}$ с образованием оксид-иона при повышенной энергии. Как и в предыдущем примере с ионами $m / z 131$, ионы с $m / z 59$ также могут иметь две структуры: структура $\mathrm{CH}_{3} \mathrm{COO}^{-}$является продуктом перегруппировочной реакции, а структура $\mathrm{CH}_{2} \mathrm{COOH}^{-}$ реализуется при простом разрыве связи.<smiles>[Y4]CC(Cc1ccccc1)C(=O)OC</smiles><smiles>[Y4]C[C@H](Cc1ccccc1)C(=O)OC</smiles>

При исследовании модифицированного дипептида каптоприла было установлено, что большинство ионов в области низких энергий образуются выбросом атомов водорода из тиоловой группы бокового заместителя фрагмента молекулы цистеина и концевой карбоксильной группы, а также в результате перегруппировочных реакций с участием этих Н-атомов. Из-за циклической структуры этого дипептида процессы простого разрыва связи происходят в области энергии свыше $4 \mathrm{eV}$, за исключением единственной реакции выделения ионов $\mathrm{SH}^{-}$. Для этого объекта на рис. 8 представлен полный ионный ток в функции от электронной энергии и кривая эффективного выхода ионов $\mathrm{c} m / z 33$.

В экспериментах с пептидами мы не обнаружили признаков их разложения на составляющие аминокислоты при взаимодействии с водой. В качестве контрольных пиков ионов в масс-спектрах дипептидов были выбраны пики с $m / z 74$ и $m / z 88$, поскольку они представляют основные ионы $[\mathrm{M}-\mathrm{H}]^{-}$в масс-спектрах глицина и аланина соответственно. В трипептидах дополнительно к ним контролировались пики с $m / z 131, m / z 145$ и $m / z 159$, поскольку они соответствуют интенсивным ионам $[\mathrm{M}-\mathrm{H}]^{-}$в масс-спектрах дипептидов, состоящих из фрагментов глицина и аланина. В диглицилаланине не были зарегистрированы ионы с $m / z 74$ и $m / z 131$, а в глицил-аланине - ионы с $m / z 74$. Другие контрольные ионы в диапазоне энергии $1-3 \mathrm{eV}$ имели низкую интенсивность и были идентифицированы как перегруппировочные ионы. Чтобы внести большую ясность в этот вопрос, мы провели эксперимент с увлажненным глицил-аланином при разных температуpax (рис. 9). Из рисунка видно, что кривая выхода ионов c $m / z 74$ в диапазоне энергии $1-3 \mathrm{eV}$ представляет 

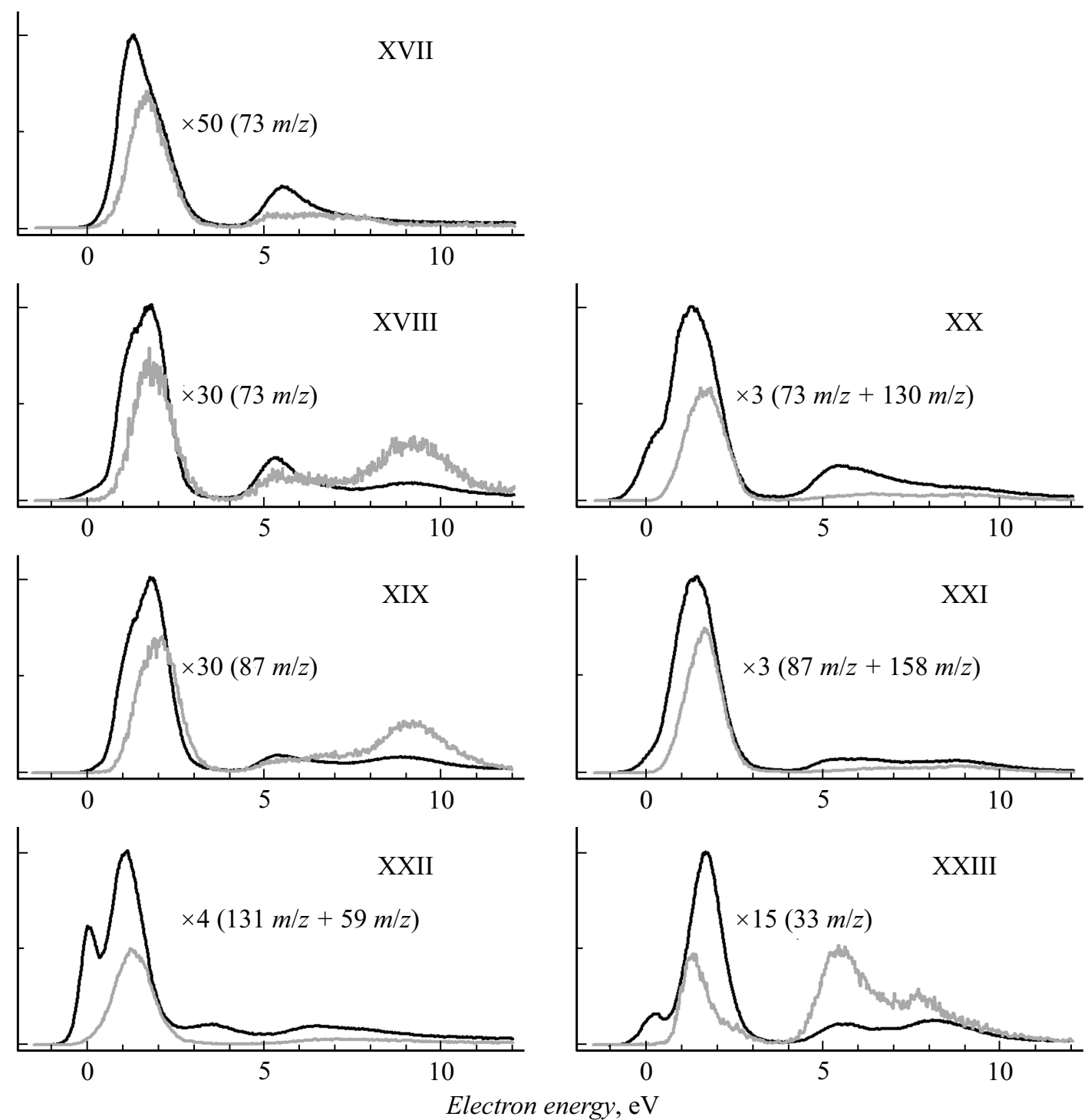

Рис. 8. Суммарные кривые эффективного выхода ОИ (черная линия) и отдельных ионов (серая линия) в зависимости от электронной энергии из пептидов при температурах: $175^{\circ} \mathrm{C}$ (диглицин), $141^{\circ} \mathrm{C}$ (глицил-аланин), $139^{\circ} \mathrm{C}$ (диаланин), $220^{\circ} \mathrm{C}$ (диглицил-аланин), $190^{\circ} \mathrm{C}$ (триаланин), $125^{\circ} \mathrm{C}$ (аспартам), $62^{\circ} \mathrm{C}$ (каптоприл).

суммарный вклад от кривых выхода изотопных пиков ионов $\mathrm{c} m / z 72$ и $m / z 73$. Это означает, что при нагреве увлажненного дипептида не происходит его разложения с образованием глицина. Кривая эффективного выхода ионов с $m / z 88$ отличается от таковой, полученной в работе [38], наличием резонансного пика в области тепловых энергий и повышенной интенсивностью пика в диапазоне энергии $1-3 \mathrm{eV}$. При увеличении температуры наблюдается уменьшение относительной интенсивности этих двух пиков, что контрастирует с результатами аналогичных экспериментов с цитидином, дезоксицитидином и дезоксиаденозином. Этот факт не позволяет связать обсуждаемые резонансные пики с образованием аланина при разложении дипептида. Возможно, что их источником являются легко летучие примеси в образце, и поэтому мы их не обнаружили в ранних длительных по времени экспериментах, включающих регистрацию масс-спектров ОИ при различных фиксированных энергиях и регистрацию кривых эффективного выхода каждого вида ионов.

Анализ полученных данных свидетельствует о том, что пептиды гораздо более устойчивы к разложению на составляющие их аминокислоты при нагреве в присутствии воды, чем нуклеозиды. Фрагментация молекулярных ОИ пептидов происходит во всем диапазоне энергии, начиная с $\sim 0 \mathrm{eV}$. Но в отличие от нуклеозидов реакции простого разрыва связей во всех исследованных пептидах протекают при энергиях свыше $1 \mathrm{eV}$. Процессы распада в области тепловых энергий происходят исключительно в результате миграции Н-атома концевой карбоксильной группы и слабосвязанных Натомов боковых заместителей. Последнее обстоятель- 

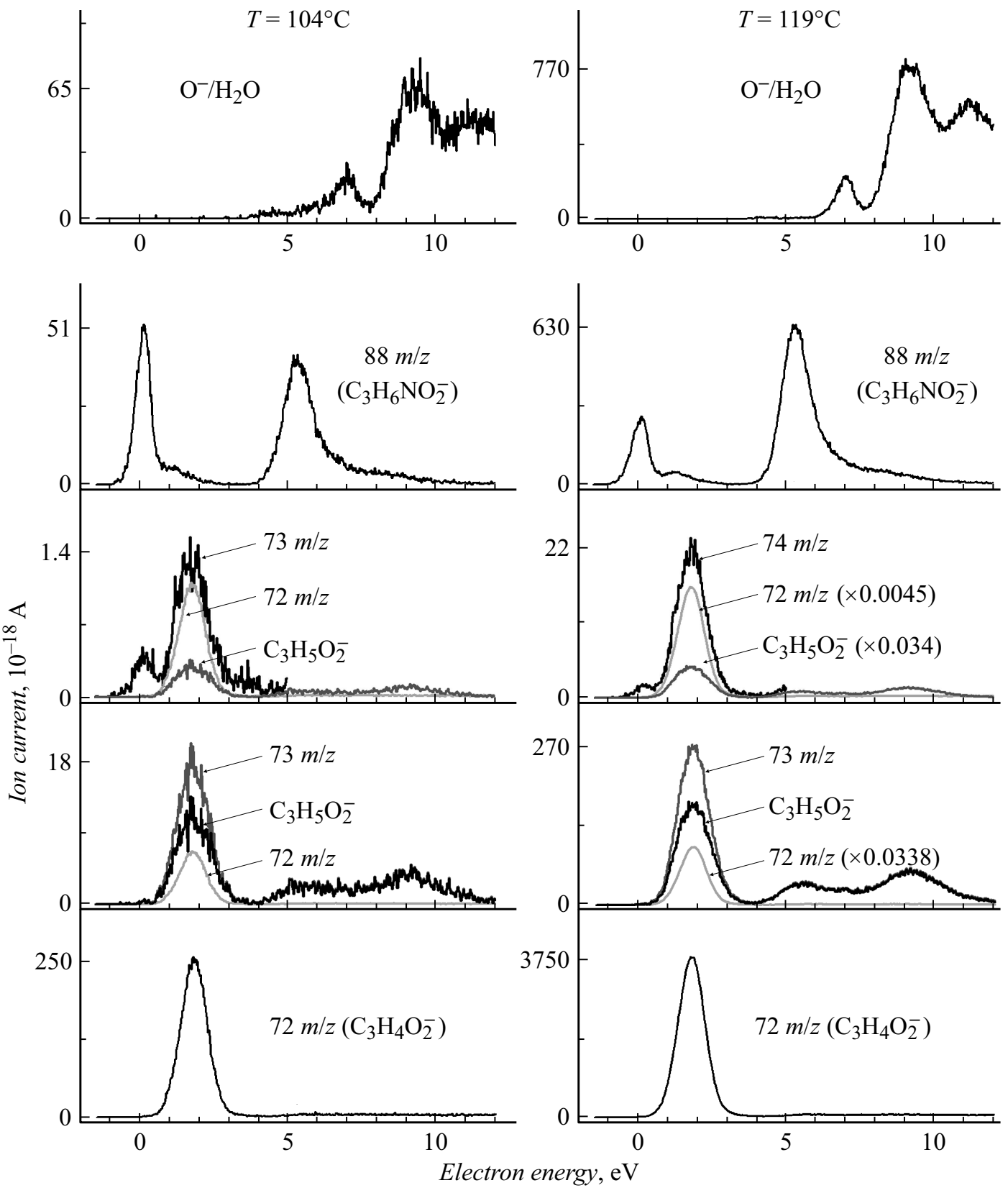

Рис. 9. Кривые эффективного выхода ионов $\mathrm{O}^{\bullet-}$ из воды (сверху) и ионов с $m / z 72, m / z 73, m / z 74, m / z 88$ из глицил-аланина (снизу) в зависимости от электронной энергии, полученные при температурах $104^{\circ} \mathrm{C}$ (слева) и $119^{\circ} \mathrm{C}$ (справа). Первая панель снизу: ионы $\mathrm{CH}_{3} \mathrm{CHCOO}^{\bullet-}(\mathrm{m} / z$ 72). Вторая панель снизу: экспериментальные кривые для пиков с $m / z 72$ (светло-серая линия) и $m / z 73$ (темно-серая линия) и как результат их разницы - кривая выхода ионов $\mathrm{CH}_{3} \mathrm{CH}_{2} \mathrm{COO}^{-}$(черная линия). Третья панель снизу: экспериментальная кривая для пика с $m / z 72$ (светло-серая линия) и математическая кривая для ионов $\mathrm{CH}_{3} \mathrm{CH}_{2} \mathrm{COO}^{-}$ (темно-серая линия) при суммировании „совпадают“ с экспериментальной кривой для пика с $m / z 74$ (черная линия). Четвертая панель снизу: ионы $\mathrm{C}_{3} \mathrm{H}_{6} \mathrm{NO}_{2}^{-}(\mathrm{m} / z$ 88).

ство препятствует использованию сложных пептидов в микроэлектронике из-за возможности их разрушения при протекании по ним электрического тока. Эта проблема может быть решена синтезом пептидов из простейших аминокислот глицина, аланина или др., не содержащих подвижных Н-атомов в боковом заместителе, и алкилзамещением подвижного Н-атома концевой карбоксильной группы (эстерификация). Тогда подача на концы такой полипептидной цепи электрического напряжения не более $1 \mathrm{~V}$ не приведет к ее распаду.

\section{Заключение}

Первые попытки измерения проводимости ДНК относятся к 60-м годам прошлого века [42], когда фактиче- 
ски была открыта ее структура, представляющая собой закрученную двойную спираль. Было установлено, что проводящие свойства нуклеиновых кислот обусловлены плотно прилегающими ароматическими гетероциклами азотистых оснований, через $\pi$-орбитали которых может осуществляться перенос электронов или дырок. C тех пор в результате многочисленных исследований электрических свойств нуклеиновых кислот был получен огромный объем информации для биомолекулярной электроники, которая на сегодняшний день является развивающимся перспективным направлением наноэлектроники. На этом фоне исследования проводимости пептидов скромнее, но, возможно, они более перспективны в качестве материалов для микроэлектроники, чем нуклеиновые кислоты. Во всяком случае, они более устойчивы к воздействию воды и низкоэнергетических электронов, что может обеспечить длительную стабильную работу электронных устройств на основе пептидов. Вторичная структура белка представляет организацию полипептидной цепи, стабилизированной водородными связями между атомом кислорода карбонильной группы и атомом водорода амидной группы, входящими в состав разных пептидных фрагментов, разделенных тремя аминокислотными остатками в случае $\alpha$-спирали и принадлежащих разным полипептидным цепям в случае складчатой $\beta$-структуры [27]. Возможно, проводящие свойства пептидов зависят от этих связей и через $\pi$ орбитали пептидных фрагментов может осуществляться перенос электронов или дырок. Для стабильной работы электронного устройства при случайных разрывах пептидной цепи выбор между $\alpha$-спиралью и складчатой $\beta$-структурой очевиден - он должен быть в пользу последней. Этот вывод основан на результатах исследований проводимости искусственно поврежденной ДНК - при разрыве одной цепи-спирали проводимость сохраняется, а при повреждении связей (через азотистые основания) между цепями-спиралями проводимость исчезает $[43,44]$. Согласно результатам работ $[45,46]$, эффективность распада нуклеиновых кислот при столкновении с низкоэнергетическими электронами носит резонансный характер, и в диапазоне энергии $\sim 0-4 \mathrm{eV}$ наблюдается одноцепочечное разрушение ДНК. Наши эксперименты показывают, что распад цитидина начинается $\mathrm{c} \sim 1 \mathrm{eV}$. Если фрагментация молекулярных ОИ гуанозина (дезоксигуанозина) также начинается с $\sim 1 \mathrm{eV}$, то электронные устройства на основе гуанинцитозиновых гомополимеров, обладающих проводимостью $p$-типа [47], могут длительно работать при напряжении на концах цепи не более $1 \mathrm{~V}$. К сожалению, эксперименты с гуанозином и дезоксигуанозином были неудачны, как и с нуклеотидами.

Работа выполнена при частичной поддержке Российского фонда фундаментальных исследований, грант № 16-08-00384_a.

\section{Список литературы}

[1] Heath J.R. // Annu. Rev. Mater. Res. 2009. Vol. 39. P. 1-23.

[2] Robertson N., McGowan C.A. // Chem. Soc. Rev. 2003. Vol. 32. P. 96.

[3] Giese B. // Bioorg. Med. Chem. 2006. Vol. 14. P. 6139.

[4] Seeman N.C. // Sci. Am. 2004. Vol. 290. P. 64.

[5] Irimia-Vladu M., Gtowacki E.D., Voss G., Bauer S., Sariciftci N.S. // Materials Today. 2012. Vol. 15. N 7-8. P. 340.

[6] Lakhno V.D. // Int. J Quant. Chem. 2008. Vol. 108. P. 1970.

[7] Мазунов В.А., Щукин П.В., Хатымов Р.В., Мубтахов М.В. // Масс-спектрометрия. 2006. Т. 3. № 1. С. 11.

[8] Muftakhov M.V., Vasil'ev Yu.V., Mazunov V.A. // Rapid Commun. Mass Spectrom. 1999. Vol. 13. P. 1104.

[9] $W u$ J., $X u$ X. // J. Chem. Phys. 2007. Vol. 127. P. 214105.

[10] Burrow P.D., Gallup G.A., Scheer A.M., Denifl S., Ptasinska S., Mark T., Scheier P. // J. Chem. Phys. 2006. Vol. 124. N 12. P. 124310.

[11] Aflatooni K., Gallup G.A., Burrow P.D. // J. Phys. Chem. 1998. Vol. 102. N 31. P. 6205-6207.

[12] Denifl S., Ptasinska S., Hanel G., Gstir B., Probst M., Scheier P., Mark T.D. // J. Chem. Phys. 2004. Vol. 120. N 14. P. 6557.

[13] Denifl S., Ptasinska S., Probst M., Hrusak J., Scheier P., Mark T.D. // J. Phys. Chem. A. 2004. Vol. 108. N 31. P. 6562 6569.

[14] Gohlke S., Abdoul-Carime H., Illenberger E. // Chem. Phys. Lett. 2003. Vol. 380. N 5-6. P. 595-599.

[15] Ptasinska S., Denifl S., Mroz B., Probst M., Grill V., Illenberger E., Scheier P., Mark T.D. // J. Chem. Phys. 2005. Vol. 123. N 12. P. 124302.

[16] Huber D., Beikircher M., Denifl S., Zappa F., Matejcik S., Bacher A., Grill V., Mark T.D., Scheier P. // J. Chem. Phys. 2006. Vol. 125. N 8. P. 084304.

[17] Hanel G., Gstir B., Denifl S., Scheier P., Probst M., Farizon B., Farizon M., Illenberger E., Mark T.D. // Phys. Rev. Lett. 2003. Vol. 90. N 18. P. 188104.

[18] Мубтахов М.В., Щукин П.В., Хатымов Р.В. // Массспектрометрия. 2013. Т. 10. № 3. С. 1-9.

[19] Ptasinska S., Denifl S., Scheier P., Mark T.D. // J. Chem. Phys. 2004. Vol. 120. N 18. P. 8505.

[20] Bald I., Kopyra J., Illenberger E. // Angew. Chem. Int. Ed. 2006. Vol. 45. N 29. P. 4851.

[21] Sulzer P., Ptasinska S., Zappa F., Mielewska B., Milosavljevic A.R., Scheier P., Mark T.D., Bald I., Gohlke S., Huels M.A., Illenberger E. // J. Chem. Phys. 2006. Vol. 125. N 4. P. 044304.

[22] Abdoul-Carime H., Gohlke S., Fischbach E., Scheike J., Illenberger E. // Chem. Phys. Lett. 2004. Vol. 387. P. 267270.

[23] Ptasinska S., Denifl S., Gohlke S., Scheier P., Illenberger E., Mark T.D. // Angew. Chem. Int. Ed. 2006. Vol. 45. P. 1893 1896.

[24] Муфтахов М.В., Щукин П.В. // Масс-спектрометрия. 2013. T. 10. № 1. С. 39-44.

[25] Бенуэлл К. Основы молекулярной спектроскопии: Пер. с англ. М.: Мир, 1985. 384 с.

[26] Bald I., Flosadóttir H.D., Omarsson B., Ingólfsson O. // Int. J. Mass Spectrom. 2012. Vol. 313. P. 15-20.

[27] Терней А. Современная органическая химия. В 2-х томах. Том 2. М.: Мир, 1981. 652 с. 
[28] Gohlke S., Rosa A., Illenberger E., Brüning F., Huels M.A. // J. Chem. Phys. 2002. Vol. 116. P. 10164.

[29] Ptasinska S., Denifl S., Candori P., Matejcik S., Scheier P., Märk T.D. // Chem. Phys. Lett. 2005. Vol. 403. P. 107.

[30] Abdoul-Carime H., Illenberger E. // Chem. Phys. Lett. 2004. Vol. 397. P. 309.

[31] Abdoul-Carime H., Gohlke S., Illenberger E. // Chem. Phys. Lett. 2005. Vol. 402. P. 497.

[32] Papp P., Urban J., Matejcik S., Stano M., Ingolfsson O. // J. Chem. Phys. 2006. Vol. 125. P. 204301.

[33] Vasil'ev Y.V., Figard B.J., Voinov V.G., Barofsky D.F., Deinzer M.L. // J. Am. Chem. Soc. 2006. V. 128. P. 5506.

[34] Kocisek J., Papp P., Mach P., Vasil'ev Y.V., Deinzer M.L., Matejcik S. // J. Phys. Chem. A. 2010. Vol. 114. P. 1677.

[35] Papp P., Shchukin P., Matejcik S. // J. Chem. Phys. 2010. Vol. 132. P. 014301.

[36] Мубтахов М.В., Щукин П.В., Хатымов Р.В., Туктаров Р.Ф. // Известия АН. Сер. Хим. 2016. № 3. С. 658.

[37] Мубтахов М.В., Щукин П.В. // Известия АН. Сер. хим. 2010. № 5. C. 875.

[38] Muftakhov M.V., Shchukin P.V. // Phys. Chem. Chem. Phys. 2011. Vol. 13. P. 4600.

[39] Shchukin P.V., Pogulay A.V., Muftakhov M.V. // Rapid Commun. Mass Spectrom. 2012. Vol. 26. N 7. P. 828.

[40] Мубтахов М.В., Щукин П.В. // Известия АН. Сер. хим. 2014. № 3. C. 642.

[41] Muftakhov M.V., Shchukin P.V. // Rapid Commun. Mass Spectrom. 2016. Vol. 30. N 24. P. 2577.

[42] Eley D.D., Spivey D.I. // Transactions of the Faraday Society. 1962. Vol. 58. P. 411.

[43] Boon E.M., Barton J.K. // Current Opinion in Structural Biology. 2002. Vol. 12. P. 320-329.

[44] Muren N.B., Olmon E.D., Barton J.K. // Phys. Chem. Chem. Phys. 2012. Vol. 14. P. 13754-13771.

[45] Boudaiffa B., Cloutier P., Hunting D., Huels M. A., Sanche L. // Science. 2000. Vol. 287. P. 1658.

[46] Sanche L. // Chem. Phys. Lett. 2009. Vol. 474. P. 1-6.

[47] Yoo K.-H., Ha D. H., Lee J.-O. // Mol. Phys. Rev. Lett. 2001. Vol. 87. P. 198102. 